Editorial

\title{
Número especial en Investigación en Psicología Ambiental
}

\section{Special Issue on Research in Environmental Psychology}

\author{
Cesar Tapia-Fonllem ${ }^{1}$ ORCID Oscar Navarro-Carrascal $^{2}$ ORCID \\ ' Universidad de Sonora, México \\ ${ }^{2}$ Universidad de Nantes, Francia
}

Forma de citar:

Tapia-Fonllem, C., \& Navarro-

Carrascal, 0. (2021). Número

especial en Investigación en

Psicología ambiental. Rev. CES

Psico, 14(1), i-iii.

\section{Open access}

(c) Copyright

Licencia creative commons

Etica de publicaciones

Revisión por pares

Gestión por Open Journal System

DOl: http://dx.doi.org/10.21615/

cesp.14.1.1

ISSN: 2011-3080

Sobre los autores:

1. Doctor en Psicología. Docente Departamento de Psicología y Ciencias de la Comunicación, Universidad de Sonora, México. Editor invitado número especial de Investigación en Psicología Ambiental de la Revista CES Psicología.

2. Doctor en Psicología. Docente Universidad de Nantes, Francia. Editor invitado número especial de

Comparte

f日E.
La agenda ambiental ha estado en el centro de las preocupaciones sociales en las últimas décadas, por los significativos efectos que el ambiente tiene sobre la salud y el bienestar humano. Es así como el estudio sobre el medio ambiente se ha convertido en un objeto de estudio científico y en blanco de interés para las instituciones encargadas de la normatividad social y el debate político.

En este orden de ideas, las relaciones de los individuos con su entorno son hoy un área estratégica de trabajo para las ciencias sociales, las ciencias de la salud y particularmente para la psicología. ¿Qué definición de medio ambiente? ¿Qué estatus epistemológico se le asigna? ¿Cuáles son las perspectivas para comprender las relaciones entre el comportamiento humano y el medio ambiente? Estas son algunas de las preguntas que busca responder la Psicología Ambiental.

El punto de partida de la Psicología Ambiental es el hecho de que la experiencia humana es en gran medida tributaria del lugar donde ocurre, es decir, depende del entorno en el que tiene lugar, de las condiciones materiales y de las normas sociales que determinan dicha relación. Es esta vivencia intuitiva la que funda la psicología ambiental, cuyo objeto de estudio se ha adaptado y evolucionado desde su nacimiento oficial a principios de la década de 1970 hasta la fecha. La psicología ambiental se define, en la actualidad, como una disciplina científica que estudia las interrelaciones entre las personas y su entorno físico y social, en referencia a las dimensiones espacial y temporal de dicha relación. El medio ambiente, objeto de percepciones, de actitudes y de comportamientos desplegados en su seno, toma su significación en relación con la dimensión temporal. La psicología ambiental hace referencia no solamente al espacio, sino también a la historia del lugar, la cual está ligada a la historia de los individuos. El medio ambiente tiene una función identitaria. Así, el marco de vida en el cual las personas viven y se desarrollan, les procura identidad en tanto las sitúa en el ámbito social, económico y cultural. La psicología ambiental se ocupa tanto de la influencia de los entornos en las experiencias humanas, como de la influencia que las personas ejercen sobre estos espacios (apropiación, territorialidad, formas de ocupación y uso). 
Investigación en Psicología Ambiental de la Revista CES Psicología.
Con el ánimo de recoger experiencias de investigación y aportes de la psicología ambiental en torno a los cambios sociales y ambientales actuales, nace este número especial. En él se presentan algunas reflexiones teóricas y metodológicas respecto a la relación de los individuos con su entorno, fruto del trabajo de investigadores de diferentes contextos institucionales y culturales. De manera especial queremos agradecer a la Universidad CES y al equipo editorial de la Revista CES Psicología por la confianza que han depositado en nosotros para coadyuvar en el presente esfuerzo académico. También destacamos la participación en el proceso de divulgación de la convocatoria a distintas organizaciones y sociedades del campo de la psicología: Asociación Colombiana de Facultades de Psicología (ASCOFAPSI): Capítulo Psicología Ambiental, Grupo de Trabajo de Psicología Ambiental de la Sociedad Interamericana de Psicología (SIP), y en Europa la Asociación de Psicología Ambiental (PSICAMB) y la Association pour la Recherche en Psychologie Environnementale (ARPEnv).

Un importante y destacado grupo de revisores de diferentes países participaron en el proceso de evaluación a doble ciego de cada artículo que recibimos, a ellos les expresamos nuestro más profundo agradecimiento por su valiosa y enriquecedora contribución. El resultado final nos permite reconocer autores y co-autores, todos ellos investigadores del campo de la Psicología Ambiental de diversos países: Colombia, Chile, Ecuador, México, España, Francia e Indonesia; y temáticas como el abordaje y estudio de las actitudes y comportamiento pro-ambientales, conducta sostenible, conectividad con la naturaleza, cosmovisión ambiental, gestión del riesgo, apego e identidad al lugar, felicidad, bienestar y futuro trascendente.

Es para nosotros un gran placer y motivo de orgullo compartir con la comunidad científica y académica de la psicología el fruto de este trabajo de varios meses. Los invitamos a consultar y utilizar los ocho artículos que conforman este número temático, presentados aquí en orden de aparición:

1. Laura Fernanda Barrera-Hernández, Víctor Corral-Verdugo y Blanca Silvia Fraijo-Sing del Instituto Tecnológico de Sonora y de la Universidad de Sonora, México, proponen el artículo El futuro trascendental como determinante de las conductas sustentables y la percepción de felicidad que busca estimar las relaciones entre dos perspectivas temporales y la conducta sustentable, y su relación con el autocuidado y la felicidad.

2. José Sebastián Sandoval Díaz, Patricio Neumann y Ricardo Rey Clericus de la Universidad del Bío Bío, Chile, presentan el articulo Adaptación y validación preliminar de la Escala teoría cultural de cosmovisiones ambientales en población chilena que busca adaptar y aportar evidencias preliminares de validez de la Escala de teoría cultural de cosmovisiones ambientales en el contexto latinoamericano.

3. Pablo Páramo y Andrea Burbano de la Universidad Pedagógica Nacional y el Colegio Mayor de Cundinamarca, Colombia, exponen el articulo Diseño de macro y metacontingencia para la promoción del comportamiento proambiental y urbano responsable, cuyo objetivo es introducir los conceptos de macrocontingencia y metacontingencia en el campo de la psicología ambiental y su papel en la promoción de acciones colectivas en pro del medio ambiente natural y construido. 
4. Pierre Dias, Raquel Bertoldo, Severin Guignard y Alexandra Schleyer-Lindenmann de la Universidad de Aix Marseille, Francia, proponen el articulo Trust in Public Risk Management and Social Dynamics in the Case of Flood Risk que busca comprender a través de un enfoque cualitativo, cómo los habitantes manejan la información oficial, y como la integran en su relación con el riesgo de inundaciones.

5. Alex Felipe Saza Quintero, Willian Sierra Barón y Cesar Andrés Gómez Acosta de la Universidad Surcolombiana y Universidad Fundación Universitaria Sanitas, Colombia, proponen el articulo Comportamiento proambiental y conocimiento ambiental en universitarios: ¿el área de conocimiento hace la diferencia?, que tiene como objetivo determinar la relación del conocimiento ambiental y el comportamiento proambiental en estudiantes universitarios y su manifestación según las áreas de conocimiento.

6. Hector Berroeta, Marcelo Rodriguez Mancilla y Marcos Zumárraga-Espinosa de la Universidad de Valparaíso, la Universidad de Playa Ancha, Chile y la Universidad Politécnica Salesiana, Ecuador, presentan el articulo Apego al Lugar e Identidad de Lugar en Barrios Patrimoniales, que busca describir y comparar los índices de Apego al Lugar e Identidad de Lugar en habitantes barrios de la ciudad de Valparaíso, Chile y de la ciudad de Quito, Ecuador, catalogados como Patrimonio de la Humanidad.

7. Laura Pasca y Juan Ignacio Aragonés de la Universidad Complutense de Madrid, España, proponen el articulo Contacto con la Naturaleza: Favoreciendo la Conectividad con la Naturaleza y el Bienestar, cuyo objetivo es conocer si el contacto con la Naturaleza se relaciona con mayores niveles de conectividad con la Naturaleza y de bienestar, en población de adolescentes y de adultos.

8. Bahrul Hayat, Rahmat Hidayat y Muhammad Dwirifai Kharisma Putra de las Syarif Hidayatullah State Islamic University, Jakarta y Gadjah Mada University, Yogyakarta, Indonesia, exponen el articulo Exploring the Factor Structure of Environmental Attitudes Measure in a Sample of Indonesian College Students, cuyo propósito es validar y evaluar la estructura factorial del instrumento de medición de actitudes hacia el medio ambiente mediante un análisis factorial y el modelo de Rasch politómico multidimensional. 